\title{
BOOTSTRAP EQUATIONS IN EFFECTIVE THEORIES
}

\section{A. V. Vereshagin, V. V. Vereshagin, and K. M. Semenov-Tian-Shansky}

In this paper, we consider general properties of effective field theories. We note that the freedom of fixing renormalization conditions in the effective field theory is not as large as it seems. Consideration of the minimal set of correctness requirements of the perturbative scheme based on the Dyson's formula for the S-matrix leads to severe restrictions on essential parameters of the theory and hence on the possible set of renormalization conditions. In the first part of this paper, we give a short review of the structure of localizable effective field theories. We discuss necessary conditions which ensure the correctness of the first step of the iterative scheme of calculation of the $S$ matrix, i.e., the construction of tree-level amplitudes. In the second part, we discuss examples which demonstrate the main stages of acquisition and analysis of the system of bootstrap equations. Bibliography: 17 titles.

\section{INTRODUCTION}

At present, two different approaches are known to the problem of constructing a relativistic quantum theory suitable to describe scattering processes. According to the first (canonical) approach, one has to fix a finite set of (fundamental) fields and a Lagrangian that satisfy certain conditions (locality, Hermiticity, symmetry, renormalizability, etc.). To obtain the quantum version, one has to carry out the procedure of canonical quantization. This allows one to construct the Fock space of asymptotic states and to calculate the Green functions and the $S$-matrix.

The second approach (suggested in [1]; we call it the $S$-matrix approach) is less known and practically has not been discussed in the literature (see, however, the works [2,3] and Chaps. $2-5$ of the monograph [4] written by the author of this approach). In this approach, the structure of the Fock space of asymptotic states is postulated initially. Field operators are constructed according to symmetry properties of the corresponding one-particle states. The Hamiltonian of the system depends on these fields and is also postulated as an operator in the interaction picture.

Elements of the $S$-matrix are calculated according to the Dyson formula ( $f$ and $i$ are the final and initial states, respectively):

$$
S_{f i}=\left\langle f\left|T_{W} \exp \left\{-i \int H_{\mathrm{int}} d x\right\}\right| i\right\rangle .
$$

The symbol $T_{W}$ in this formula denotes the Wick's (obviously covariant) $T$-product. Noncovariant terms in the Hamiltonian and in propagators are to be discarded (see [4]). In the case of effective theories (only such theories are discussed below), this does not lead to any uncertainties.

Each of these two approaches has its advantages and shortcomings. However, their comparative analysis is not our goal here. We use the $S$-matrix approach due to the following reason: canonical quantization of theories with Lagrangians containing high (second and higher) powers of field time derivatives is a problem for which no solution is presently known. At the same time, effective theories, which are the main subject of our study, contain all of the powers of first (and higher) field derivatives by the construction itself.

We use a slightly improved version of a definition of an effective theory (originally given in [5]): a theory is called effective if the corresponding Hamiltonian (in the interaction picture) contains all of local terms consistent with the requirements of the given algebraic symmetry. In the operator sense, this construction is not well defined. However, we are only interested in $S$-matrix elements calculated (only on the mass shell!) with the help of the expansion based on formula (1). In the works $[6,7]$, it was shown that it is possible to formulate for these objects simple correctness conditions for expressions calculated in a given order of loop expansion. In the zero order (tree graphs) of the renormalized perturbation theory, these conditions lead to reasonable restrictions on the values of physical parameters of a theory.

Effective theories, by their construction, indicate the property of multiplicative renormalizability (in the case of absence of anomalies). However, usually they are not considered in textbooks on the renormalization theory (see, for example, [8]). The reason is as follows: to fix the physical content of such a theory, one has to

Translated from Zapiski Nauchnykh Seminarov POMI, Vol. 291, 2002, pp. 78-108. Original article submitted November 18, 2002. 
impose an infinite set of renormalization prescriptions (conditions). This is absolutely unrealistic if we have no leading principle limiting the freedom of this step. Preliminary results of our research (see [6,7]) show that the analyticity-type restrictions could play the role of such a principle. In addition, these restrictions seem to be natural from the point of view of correctness of the perturbative scheme. For example, it is possible to obtain the bootstrap conditions (widely discussed in connection with dual models, see [9]) from properly formulated requirements of meromorphy and polynomial boundedness of the tree-level amplitudes constructed in the framework of the renormalized perturbation theory.

In this paper, we illustrate the techniques of derivation of the bootstrap equations from the condition that a function of two complex variables is meromorphic and polynomially bounded in various domains. However, before starting the analysis of examples, we would like to outline the reasons for which these conditions (automatically fulfilled in conventional field theories) turn out to be fruitful in the case of an effective theory. The following three sections serve this purpose.

\section{Preliminary REMARKS}

First of all, we recall the definitions of some notions and terms used below. We specify the definitions given in [4] and introduce the notion of minimal parametrization of an effective theory. We introduce these notions since they are suitable in the work with on-shell matrix elements in terms of complex analysis.

Let us emphasize that in what follows it is assumed that the masses of particles with spin $J>1 / 2$ are nonzero. This assumption is purely technical, but at present we cannot proceed without it. It does not lead to any limitations when we are describing strong interaction of hadrons.

All independent combinations of coupling constants which do not appear in expressions for the renormalized $S$ matrix elements of $L$ th order in loop expansion are called redundant parameters of order $L$. These combinations may appear in expressions for the Green functions, but the corresponding contributions turn into zero after renormalization, passage to the mass shell, and multiplication by relevant wave functions. An example of a redundant parameter is given by the gauge fixing constant in renormalizable vector models. Another example is the wave function renormalization constant [4].

All independent combinations of coupling constants that appear in expressions for the renormalized matrix elements of $L$ th order of the loop expansion are called essential parameters of order $L$.

Consider an elementary (pointlike) vertex with $n$ legs carrying momenta $p_{1}, p_{2}, \ldots, p_{n}$. In general, this vertex can be represented as follows:

$$
V\left(p_{1}, \ldots, p_{n}\right)=\sum_{a=1}^{M+N} T^{(a)} F_{a},
$$

where $\left\{T^{(a)}\right\}$ is the complete set of independent tensor structures ( $M$ of these structures are minimal and $N$ are nonminimal; see the definitions below) and $F_{a}$ are functions of invariant kinematic variables (the total number of these variables is equal to 3 if $n=3$ and to $4 n-10$ if $n>3$; we work in $D=3+1$ dimensions). It is convenient to choose these variables as follows:

$$
[\pi, \nu] \equiv\left[\pi_{1}, \ldots, \pi_{n} ; \nu_{1}, \ldots, \nu_{3 n-10}\right]
$$

where

$$
\pi_{i} \equiv p_{i}^{2}-m_{i}^{2}
$$

A concrete choice of the remaining $3 n-10$ variables $\nu_{i}$ (independent linear combinations of momentum scalar products) is not important for present.

Vertex (2) is an element of the system of Feynman rules of the effective theory under consideration. This vertex describes the joint contribution of terms in the Hamiltonian which are constructed from $n$ field operators.

It is clear that the functions $F_{a}$ are polynomial in variables $(3)$; coefficients of these polynomials are combinations of coupling constants. It would be premature to discuss convergency conditions for series of items corresponding to vertices with various number of derivatives. Our purpose is to obtain well-defined expressions for the $S$-matrix elements of given order; to obtain such expressions, one has to take into account not only vertices of type (2) but also the contributions of all possible graphs with $n$ legs. In what follows, we only care about the correctness of expressions which appear as a result of infinite summation of graphs.

The contribution of vertex (2) to the matrix element describing the process with $n$ external particles can be obtained by the passage to the mass shell $\pi_{i}=0(i=1, \ldots, n)$ and multiplication by relevant wave functions. Hence, the combinations of coupling constants which form coefficients at nonzero powers of $\pi_{i}$ in the series for 
$F_{a}$ do not appear in the contribution of the corresponding pointlike vertex to the $S$-matrix elements in question. The same is true for combinations of coupling constants which appear in expressions for the functions $F_{a}$ that are coefficients at tensor structures vanishing after the passage to the mass shell and multiplication by relevant wave functions (such structures are called nonminimal).

It is easy to understand that the same is true for vertices with an arbitrary number of "bubbles" (self-closed lines) and "tadpoles" (lines starting at a vertex and ending by one or several "bubbles"). We treat such vertices as pointlike.

Thus, after putting vertex (2) to the mass shell and multiplication by relevant wave functions, this vertex takes the following form:

$$
V\left(p_{1}, \ldots, p_{n}\right) \stackrel{\text { onshell }}{\sim} \sum_{a=1}^{M} T^{(a)} F_{a}(\pi=0, \nu) .
$$

Here the set of $T^{(a)}(a=1, \ldots, M)$ contains only minimal tensor structures and does not contain nonminimal ones.

The line $p_{k}$ of a vertex $V\left(p_{1}, \ldots, p_{n}\right)$ is called minimal if the explicit form of expression (2) is not changed when the $k$ th particle is put on its mass shell $\pi_{k}=0$ and the vertex is multiplied by the relevant wave function $u\left(p_{k}\right)$.

We say that a vertex $V\left(p_{1}, \ldots, p_{n}\right)$ is minimal if all of its lines are minimal. A minimal vertex can always be presented in the form (5). Finally we say that the propagator of a particle with mass $m$ and spin $J$ is minimal if its numerator is precisely its spin sum written in a covariant form (note that $q^{2}=m^{2}$ on the mass shell!) and considered as a function of four independent components of the momentum. ${ }^{1}$

Each $S$-matrix graph of an effective theory can be rewritten in terms of minimal elements, vertices (of different orders), and propagators (see [10]; a complete proof will be published later). This means that the full set of essential parameters includes only masses and the combinations of coupling constants which define the minimal parametrization of vertices of different orders. ${ }^{2}$ Thus, to obtain a finite $S$-matrix it is sufficient to impose only those renormalization prescriptions which are necessary to fix finite parts of minimal parameters. However, the results of the works $[6,7]$ show that this set of prescriptions is also excessive. The necessity of following the bootstrap restrictions (see below) results in the fact that only a part of minimal parameters should be treated as independent constants.

Renormalization prescriptions should only be imposed on the set of independent constants of the theory. This means that there are two (equivalent) methods to impose them. The first method consists in finding an explicit solution of the bootstrap conditions. ${ }^{3}$ Following this method, one singles out the full set of independent parameters and then imposes renormalization prescriptions for this set. The second method is to impose an arbitrary set of prescriptions on all of minimal parameters and then to use the bootstrap equations as binding limitations on the possible structure of this set. We use the second method (just because we cannot find an explicit solution of the bootstrap conditions); thus, we want to discuss it in more detail.

Renormalization prescriptions, irrespectively to their explicit form, are to be satisfied at every fixed order. In particular, this is true for the lowest (tree-level) order. This means that each relation between minimal parameters of zero order (recall that in the framework of the renormalized perturbation theory, this is a relation between the physical values of the parameters!) has to be treated as a relation between prescriptions. In other words, if there exist some connections between minimal parameters of zero order (this is exactly the case for mathematically reasonable effective theories), then one cannot impose arbitrary renormalization prescriptions. This statement would be trivial if we were discussing restrictions due to some symmetry (group) limitations. But in the case of effective theories these restrictions (bootstrap equations) arise from certain requirements of localizability, which are discussed in the following section.

\section{LocAlizABle EFFECTIVE THEORIES}

In the case of an ordinary renormalizable theory, every term of the loop expansion of the $S$-matrix based on expression (1) is well defined (regularization is implied). The convergence problem for this expansion remains open, but this does not create obstructions at an arbitrary finite order. The situation is quite different in the

\footnotetext{
${ }^{1}$ This is precisely the point at which our assumption on the absence of massless particles of higher $(J>1 / 2)$ spin becomes important.

${ }^{2}$ An explicit expressions of minimal parameters in terms of coupling constants certainly depends on the perturbation order.

${ }^{3}$ Such a solution certainly exists; an example is provided by conventionally renormalizable theories.
} 
case of an effective theory. The Hamiltonian contains terms with many derivatives (of arbitrarily high degree and order), hence infinite power series appear in expressions for matrix elements already at the tree level. In other words, in this case the Hamiltonian is not a local operator, and one has to be cautious working with it. For this reason, in what follows we restrict ourselves to a special class of effective theories.

We only consider Hamiltonians of localizable type. Intuitively, one can present the idea of localizability considering a simple example from electrostatics. The interaction Hamiltonian of a point charge with an extended charge is nonlocal. Nevertheless, under certain conditions (for well separated systems), the Hamiltonian can be localized (written in the form of a convergent infinite series of local terms) with the help of a multipole expansion.

Localizability requirements can be briefly formulated in an implicit form. The explicit formulation would take too much space without real illuminating the general idea. The main idea is suggested by the quasiparticle method [11], which is well known in the nonrelativistic quantum mechanics.

We call a Hamiltonian localizable if the tree-level amplitudes formally obtained from it can be reproduced in the framework of a well-defined tree approximation of a certain extended effective theory containing auxiliary fields corresponding to particles (with masses $M_{i}$ ) that are unstable with respect to decays into states of the initial theory.

An important remark: in the instability condition

$$
M_{i} \geq m+\mu
$$

small letters denote physical particle masses, i.e., the masses of asymptotic states of the Hamiltonian of the initial theory (see [4]). The quantities $M_{i}$ have the meaning of mass parameters of the extended Hamiltonian. They define positions of the poles of bare propagators. Thus, their treatment depends on the applied renormalization scheme (see $[12,13])$. However, a detailed study of this problem lies beyond the scope of this paper. We use the term "mass" in both senses since this leads to no misunderstanding.

The words "well-defined" are to be understood in the following sense: the formal tree level series of the extended theory must be summable in the whole domain of definition. In addition, the tree level series of the initial theory must converge at least in a small domain $D$; otherwise, no comparison is impossible. In this domain, the tree approximations for amplitudes describing scattering and creation of stable particles in both theories must coincide identically. In other words, in the sector of stable particles, the tree approximation of the extended theory is precisely the analytic continuation of the tree approximation of the initial theory. This is the essence of the extension idea. Condition (6) is necessary to ensure the coincidence of asymptotic states in both theories. In the extended theory, loop corrections lead to instability of particles described by auxiliary fields, and the asymptotic space (the space of stable states) coincides with that in the initial theory. It is well known (see [14]) that in the theory with unstable particles, the $S$-matrix constructed in accordance with the formal Feynman rules is a unitary operator on the space of stable states.

Before transforming this verbal description of localizable theories into definite limitations on values of coupling constants, we have to make several preliminary remarks.

The requirement of localizability reflects a natural wish to work with series such that each term is a welldefined function of momenta. In addition, by the construction itself, the general structure of formula (1) provides a guarantee of covariance, causality, unitarity, and crossing symmetry of the $S$-matrix.

At present, the only known tool producing series with the desired structure is the formalism of the quantum field theory with a Hamiltonian containing a finite number of local interaction terms. It is essential that in this approach, the tree level amplitude of every process is a rational function of any pair energy (with all other variables fixed). It is easy to see that in the case of a theory containing an infinite number of scalar fields $\phi_{i}$ $(i=1,2, \ldots)$ with masses $m_{i}$ and the renormalizable type of interaction,

$$
H_{\mathrm{int}}=g_{i j k} \phi_{i} \phi_{j} \phi_{k}+\lambda_{i j k l} \phi_{i} \phi_{j} \phi_{k} \phi_{l}
$$

no changes are needed in the general scheme of quantum theory. It is sufficient that the matrices of coupling constants $g_{i j k}$ and $\lambda_{i j k l}$ and the mass vector $m_{i}^{2}$ satisfy conditions ensuring the convergence of series at every given order of loop expansion. Under this condition, the tree-level amplitudes of all processes (not only of those describing scattering and production of stable states) are meromorphic functions of any pair energy. In addition, one has to require (see $[7,10]$ ) that these functions are polynomially bounded (in the sense of contour asymptotic, see, for example, [15]) at zero momentum transfer.

This condition is necessary if we want to construct loop graphs by closing external lines in the corresponding tree graphs, being free in the order of operations. 
In principle, the situation in the initial effective theory does not differ from that described above if the analytically continued (from the postulated convergency domain $D$ ) amplitudes are polynomially bounded meromorphic functions (not arbitrary, see below) of any pair energy. The meromorphy properties give us a hope that these functions can be reproduced in the framework of the extended effective theory containing auxiliary fields with suitable masses. Of course, this is not always possible since not any meromorphic function can be obtained as a tree-level amplitude of some hypothetical field theory. Thus, the requirements of localizability are to be treated as conditions of the existence of an extended effective theory of the most general form. The only restriction is that the mass spectrum of the latter theory must satisfy the instability condition (6). We call these requirements analyticity conditions. This special term is used to avoid the necessity of emphasizing the formal difference between the Hamiltonians of the extended and initial theories. The extended theory is introduced precisely because we do not have a tool allowing us to work beyond the frames of the Dyson's perturbation theory.

Restrictions on coupling constants of the extended theory can be transformed into the desired conditions of localizability restricting the possible set of couplings in the initial Hamiltonian. For this purpose, it is sufficient to compare the expansions of scattering amplitudes calculated in both theories in the domain $D$.

\section{Analyticity COnditions}

First of all, we have to consider the extended theory and formulate the analyticity conditions. However, this problem is rather complicated. In this paper, we make only the first step: we formulate (necessary) analyticity conditions for the amplitudes of binary processes. This case is relatively simple since the kinematics is described completely by two independent variables.

It is convenient to introduce the following three equivalent sets:

$$
\left(x, \nu_{x}\right), \quad x=(s, t, u),
$$

where $(s, t, u)$ are the conventional Mandelstam variables, and

$$
\nu_{s}=t-u, \quad \nu_{t}=u-s, \quad \nu_{u}=s-t .
$$

The tree-level amplitude $M(s, t, u)$ of an arbitrary binary process with scalar particles (the generalization to the case of arbitrary spins does not lead to any particular difficulties) constructed in accordance with the Feynman rules takes the form of the following formal series:

$$
M(s, t, u)=\sum_{i, j, k=0}^{\infty} a_{i j k} s^{i} t^{j} u^{k}+\sum_{R_{s}} \frac{N_{s}(s, t, u)}{s-M_{R}^{2}}+\sum_{R_{t}} \frac{N_{t}(s, t, u)}{t-M_{R}^{2}}+\sum_{R_{u}} \frac{N_{u}(s, t, u)}{u-M_{R}^{2}} .
$$

The summation is taken over all kinematically allowed resonances $R_{x}\left(M_{R}\right.$ stands for the mass of the corresponding resonance) in each channel and also (in the first sum) over all four-particle vertices. In its turn, the numerators $N_{x}(s, t, u)$ take the form of (formal) infinite sums:

$$
N_{x}(s, t, u)=\sum_{i, j, k=0}^{\infty} b_{i j k}^{(x)} s^{i} t^{j} u^{k}
$$

Numerical matrices $a_{i j k}$ and $b_{i j k}^{(x)}$ are functions of coupling constants of the (extended) Hamiltonian.

Series (9) must be summable to make sense and to be used for constructing next orders of the loop expansion. The result must be a meromorphic function in each of the Mandelstam variables. To provide the possibility of constructing loops and performing the renormalization procedure, this function (of one complex variable $\nu_{x}$ ) should necessarily ${ }^{4}$ be polynomially bounded at $x=0$ and, by continuity, in a small vicinity of this value.

With the help of definitions (8), one can rewrite the formal series (9) in three different forms:

$$
\begin{aligned}
& M(s, t, u)=\sum_{i=0}^{\infty} \alpha_{i}^{(s)}(s) \nu_{s}^{i}+\sum_{R_{t}} \frac{\rho^{(s t)}(s)}{\left(\sigma-2 M_{R}^{2}\right)-s+\nu_{s}}+\sum_{R_{u}} \frac{\rho^{(s u)}(s)}{\left(\sigma-2 M_{R}^{2}\right)-s-\nu_{s}}, \\
& M(s, t, u)=\sum_{i=0}^{\infty} \alpha_{i}^{(t)}(t) \nu_{t}^{i}+\sum_{R_{u}} \frac{\rho^{(t u)}(t)}{\left(\sigma-2 M_{R}^{2}\right)-t+\nu_{t}}+\sum_{R_{s}} \frac{\rho^{(t s)}(t)}{\left(\sigma-2 M_{R}^{2}\right)-t-\nu_{t}}
\end{aligned}
$$

${ }^{4}$ One can understand this considering the loop graph as the integral of the product of a tree graph and a relevant propagator. The integral is taken at zero momentum transfer between the legs which are being closed [10]. 
and

$$
M(s, t, u)=\sum_{i=0}^{\infty} \alpha_{i}^{(u)}(u) \nu_{u}^{i}+\sum_{R_{s}} \frac{\rho^{(u s)}(u)}{\left(\sigma-2 M_{R}^{2}\right)-u+\nu_{u}}+\sum_{R_{t}} \frac{\rho^{(u t)}(u)}{\left(\sigma-2 M_{R}^{2}\right)-u-\nu_{u}} .
$$

Here $\sigma$ stands for the sum of squares of the masses of external particles. The right-hand sides of expressions (10)-(12) are written in terms of the natural coordinate systems in the corresponding layers:

$$
B_{x}\left\{x \in \mathbf{R}, x \sim 0 ; \nu_{x} \in \mathbf{C},\left|\nu_{x}\right|<\infty\right\}, \quad x=(s, t, u)
$$

This form of notation is convenient for constructive formulating of analyticity conditions. Note that each pair of layers (13) has a nonempty intersection:

$$
D_{s}=B_{t} \cap B_{u}, \quad D_{t}=B_{u} \cap B_{s}, \quad \text { and } \quad D_{u}=B_{s} \cap B_{t} .
$$

Hence, the summability conditions in every layer are to be adjusted so that in the domain

$$
R=B_{s} \cup B_{t} \cup B_{u},
$$

these conditions define a unique meromorphic function. The system of bootstrap equations is an algebraic form of these matching conditions.

Taking into account the quoted above general considerations, we formulate the analyticity conditions for the amplitudes of binary processes as follows ${ }^{5}[7,10]$. The tree-level amplitudes must be meromorphic functions in each pair energy $s_{i j} \in \mathbf{C}$ at arbitrary fixed ${ }^{6}$ value of the second independent variable. In every layer (13) containing the zero-value hyperplane of one of the momentum transfers $x$, these amplitudes must be polynomially bounded functions of the corresponding variable $\nu_{x}$. The bounding polynomial degree $N$ may depend on the quantum numbers characterizing the process.

This formulation of analyticity conditions might seem unnecessarily complicated, especially if one takes into account that domain (15) is only a part of the full complex space of two variables describing the process. We use this formulation for the following two reasons. First, the results of the works $[6,7]$ and $[10]$ show that the above formulation leads to reasonable physical consequences. Second, even in the case under consideration (binary processes), the corresponding systems of bootstrap equations for minimal parameters of the extended theory are very complicated. In this case, an imprudent attempt to formulate more general requirements without sufficient physical and mathematical motivation can lead to inconsistency.

The examples which we analyze in the following sections illustrate the structure and techniques of derivation of bootstrap equations. Before starting such considerations, we make a short review of the Cauchy form method.

\section{CAUChY FORMS}

We apply a method (known from complex analysis; see, e.g., [15]) which allows us to present a polynomially bounded meromorphic function of one complex variable as a uniformly converging series of pole contributions (in what follows, we call such representations Cauchy forms or Cauchy expansions). The possibility of working in a layer

$$
B_{x}\{x \in \mathbf{R}, x \in(a, b) ; z \in \mathbf{C},|z|<\infty\}
$$

(and not only in a plane $x=$ const) is provided by the following natural modification of the method (see [6, 7]): all of the coefficients are assumed to be smooth (real-analytic) functions of the parameter $x$.

First of all, we have to specify the definition of the bounding polynomial degree; this is important for the analysis of effective theories. We assume that the reader is familiar with the notion of a system of contours $C_{n}$ which appears in the definition of a polynomially bounded meromorphic function of one complex variable (see [15]). Working in layer (16), we assume a smooth dependence of this system on the parameter $x$. A meromorfic function $f(x, z)$ defined in layer (16) is called polynomially bounded with degree $N$ (or, simply $N$-bounded) if $N$ is the minimal integer such that

$$
\left.\frac{|f(x, z)|}{z^{N+1}}\right|_{z \in C_{m}} \stackrel{m \rightarrow \infty}{\longrightarrow} 0
$$

\footnotetext{
${ }^{5}$ Let us emphasize that here we only discuss scalar amplitudes (the functions $F^{(a)}$ in (5)).

${ }^{6}$ Of course, in the domain of definition.
} 
for all $x \in(a, b)$. The Cauchy form allows one to represent a function $f(x, z)$ that is $N$-bounded in layer (16) as a uniformly convergent series of pole contributions. For our further purposes, we are mostly interested in the case where the poles are simple and there is no pole at $z=0$. In this case, the Cauchy form looks as follows:

$$
f(x, z)=\sum_{n=0}^{N} \frac{1}{n !} f^{(n)}(x, 0) z^{n}+\sum_{i=1}^{\infty}\left\{\frac{r_{i}(x)}{z-p_{i}(x)}-h_{i}^{(N)}(x, z)\right\} .
$$

Here $p_{i}(x)$ and $r_{i}(x)$ are the position of $i$ th pole and the corresponding residue. The poles are numbered so that

$$
\left|p_{i}(x)\right| \leq\left|p_{i+1}(x)\right|
$$

The correcting polynomials $h_{i}^{(N)}(x, z)$, which ensure the convergence of the series, look as follows:

$$
h_{i}^{(N)}(x, z) \equiv-\frac{r_{i}(x)}{p_{i}(x)} \sum_{n=0}^{N}\left[\frac{z}{p_{i}(x)}\right]^{n} .
$$

It is not difficult to show [7] that for an $N$-bounded function $f(x, z)$, which is represented by formula (18) in layer (16), certain "collapsing" conditions are valid: the series of correcting polynomials of higher orders $M>N$ converge to the values of the corresponding derivatives:

$$
\sum_{i=1}^{\infty} h_{i}^{(N+k)}(x, 0)=\frac{1}{(N+k) !} f^{(N+k)}(x, 0), \quad x \in(a, b) \quad k=1,2, \ldots .
$$

These conditions help us to puzzle out the system of bootstrap equations; a corresponding example is given below.

The form (18) is the main tool used in $[7,10]$ to derive the bootstrap equations.

\section{Bootstrap EquATions: A SIMPle EXAMPLE}

Consider a simple example allowing us to illuminate the general scheme discussed in the previous sections. This example allows us to demonstrate explicitly the method of deduction as well as the structure of the bootstrap equations for parameters of a rational function of two variables restricted by the corresponding analyticity conditions. This example admits an explicit solution and makes the terminology more transparent.

Consider a rational function $F(x, y)$ of two complex variables. Assume (this is an analog of analyticity requirements) that in the layer

$$
B_{y}\{x \in \mathbf{C} ; y \in \mathbf{R}, y \in(-\eta,+\eta)\},
$$

the function has a single pole (in $x$ ) and in the layer

$$
B_{x}\{y \in \mathbf{C} ; x \in \mathbf{R}, x \in(-\xi,+\xi)\}
$$

it also has a single pole (in $y$ ). We consider decreasing asymptotics in any of the layers (in terms of Sec. 5, the considered function is 0-bounded in any of the layers). We search for an answer to the following question: what is the structure of the set of essential parameters describing this function?

In this case, essential parameters are precisely the coefficients $f_{i j}$ of the expansion

$$
F(x, y)=\sum_{i, j=1}^{\infty} f_{i j} x^{i} y^{j}
$$

The question above can be reformulated in a more concrete way: how many independent combinations can be fixed arbitrarily and what are these combinations? In terms of the field theory, the question is: how many independent renormalisation prescriptions is it necessary to impose to fix the amplitude $F(x, y)$ in a unique way and what is the explicit form of those prescriptions?

In layer (21), the function $F(x, y)$ can be represented as follows:

$$
F(x, y)=\frac{\rho(y)}{x-\pi(y)}, \quad(x, y) \in B_{y}
$$


The functions $\rho(y)$ and $\pi(y)$ are assumed to be smooth in a vicinity of the origin:

$$
\pi(y)=\sum_{i=0} \pi_{i} y^{i} \quad \text { and } \quad \rho(y)=\sum_{i=0} \rho_{i} y^{i} .
$$

Similarly, in layer (22),

$$
F(x, y)=\frac{r(x)}{y-p(x)}, \quad(x, y) \in B_{y}
$$

where

$$
p(x)=\sum_{i=0} p_{i} x^{i} \quad \text { and } \quad r(x)=\sum_{i=0} r_{i} x^{i}
$$

In the domain

$$
D_{x y}=B_{x} \cap B_{y}
$$

we obtain the following equality:

$$
\frac{r(x)}{y-p(x)}=\frac{\rho(y)}{x-\pi(y)}, \quad(x, y) \in D_{x y} .
$$

Substituting series (25) and (27) into formula (28), we obtain an infinite system of conditions on the coefficients $p_{k}, r_{k}, \pi_{k}, \rho_{k}$ :

$$
r_{i+1} \pi_{0}-p_{i+1} \rho_{0}=r_{i}, \quad \rho_{i+1} p_{0}-\pi_{i+1} r_{0}=\rho_{i}, \quad \text { and } \quad r_{i+1} p_{j+1}=\rho_{i+1} \pi_{j+1}, \quad i, j=0,1, \ldots
$$

This system provides an example of what is called the bootstrap equations in [7]. Once solved, this system allows us to express the parameters $p_{i}, r_{i}$ in terms of $\pi_{i}, \rho_{i}$. It also gives an answer to the following question: is it possible to perform an analytic continuation from one layer to another. System (29) is an infinite system of equations with $2 \times \infty$ (formal notation) unknown parameters, which we need to express the function $F(x, y)$ in layer (22) in terms of the parameters defining it in layer (21). In general, it is very difficult to find solutions of such systems and even to establish their solvability. Fortunately, in this simple example it is possible to find an explicit form of the solution. This exercise is really useful since it demonstrates the "power" of bootstrap restrictions. Rewriting condition (28) as

$$
r(x)[x-\pi(y)]=\rho(y)[y-p(x)]
$$

taking the second derivatives $\partial_{x y}^{2}$, and separating the variables, we obtain the relation

$$
\frac{r^{\prime}(x)}{p^{\prime}(x)}=\frac{\rho^{\prime}(y)}{\pi^{\prime}(y)} \equiv a,
$$

where primes mean derivatives with respect to the corresponding variables and $a$ is the separation parameter. From relation (31) we obtain the expressions

$$
r(x)=a p(x)+b \quad \text { and } \quad \rho(y)=a \pi(y)+c,
$$

where $b$ and $c$ are new constants. Finally, substituting expressions (32) into (30) and separating the variables once more, we get the equalities

$$
p(x)=\frac{d-b x}{c+a x} \quad \text { and } \quad \pi(y)=\frac{d-c y}{b+a y},
$$

were $d$ is another separation parameter. Formulas (33) combined with relations (32), (24), and (26) give us an exhaustive solution of the problem (it is easy to check that the exceptional cases give no solutions). The important property of this solution is that it contains only four arbitrary parameters. This means that the infinite system (29) is consistent only if the function $F(x, y)$ defined in layer $(21)$ belongs to the four-parametric family

$$
F(x, y)=\frac{a d+b c}{-d+a x y+b x+c y} .
$$


This is the only case where there exists an analytic continuation of this function from $B_{y}$ into $B_{x}$ with the desired properties. It is clear that in this case this continuation is unique.

Direct analysis of system (29) would lead to the same conclusion. Such an analysis is possible in this simple example. Unfortunately, no regular methods for solving infinite-dimensional algebraic systems are known, with exception of some trivial cases.

Formula (34) allows us to express the essential parameters,

$$
f_{i j}=f_{i j}(a, b, c, d)
$$

in terms of the "fundamental constants" $(a, b, c, d)$. One can choose four arbitrary coefficients $f_{k}(k=1,2,3,4)^{7}$ allowing the inversion,

$$
a=a\left(f_{1}, \ldots, f_{4}\right), \quad \ldots, \quad d=d\left(f_{1}, \ldots, f_{4}\right),
$$

and impose arbitrary "renormalization conditions" for these four quantities. Renormalization of the remaining essential parameters has to respect conditions (29).

Thus, now we can answer the question posed in the beginning of this section. To fix the amplitude $F(x, y)$ uniquely, it is sufficient to impose four renormalization prescriptions fixing the "fundamental" constants a, $b, c, d$.

This example explains the prudence with which we have formulated analyticity conditions in Sec. 4. If, in addition to these conditions, one imposes supplementary analyticity conditions (for example, in the layer

$$
C_{x}\{y \in \mathbf{C} ; x \in \mathbf{R}, x \in(1-\xi, 1+\xi)\}
$$

with an arbitrary number of poles and arbitrary asymptotic in this layer), then, with the exception of a lucky chance, one would come to a contradiction.

It is interesting to note that if we modify the problem and demand that the function $F(x, y)$ has one pole in the layer $B_{x}$ (as in the previous case) but is 1-bounded (instead of 0-bounded) in this layer, we would obtain a solution also depending on four parameters. This solution, however, would be found among the exceptional cases.

\section{CAUChy FORMS FOR THE STRING AMPLitude}

The example considered in Sec. 6 was too simple, and the method, which was applied to solve it, can hardly be useful in the case of effective theories where the number of poles is infinite. In this section, we show how to obtain the bootstrap conditions for a function with an infinite number of poles. For this puprose, we use the technique of Cauchy forms. Of course, we cannot find explicit solutions of those conditions. However, we show that even if a function $F(x, y)$ is given explicitly (i.e., the set of minimal parameters is known), the bootstrap conditions can serve as a source of nontrivial relations connecting the parameters. Precisely this property has been used in $[7,10]$ to obtain restrictions on physical characteristics of pion-kaon and pion-nucleon scattering processes.

As an illustrative example, we have chosen the Euler $B$-function (or, to be more precise, the so-called Lovelace amplitude [16], which differs by a factor). This choice is explained by several reasons. First of all, it is easy to follow the details of calculations, since all the necessary identities are widely known. Second, in spite of the existence of a lot of summation formulas for the Pochhammer symbols, we obtain (using a very simple and extremely elegant method) an infinite sequence of identities which it would be hard to deduce by traditional methods. Third, the Euler $B$-function plays an important role in dual models and in string theory (see [9]). Thus, our choice is justified from the physical point of view. Finally, the last argument in favor of our choice is that numerical tests of the corresponding bootstrap relations allow us to understand qualitatively the structure of criteria which are necessary to evaluate the rate of convergence. The latter point becomes very important when one tries to compare various theoretical predictions (sum rules) with experimental data.

Let us consider a simple (string-like) model for the scattering amplitude, which is constructed (in accordance with the idea of Veneziano [17]) from the $B$-function without a tachyon:

$$
A(s, t)=(-s-t) B\left(\frac{1}{2}-s, \frac{1}{2}-t\right)=\frac{\Gamma\left(\frac{1}{2}-s\right) \Gamma\left(\frac{1}{2}-t\right)}{\Gamma(-s-t)} .
$$

\footnotetext{
${ }^{7}$ Or four arbitrary combinations.
} 
This function has the following specific points (hyperplanes) $(m, n=0,1,2, \ldots)$.

- Zero hyperplanes: $s+t=n$.

- Pole hyperplanes in $s$ ( $t$ fixed, $s+t \neq m): s=\frac{1}{2}+n$.

- Pole hyperplanes in $t$ ( $s$ fixed, $s+t \neq m): t=\frac{1}{2}+n$.

- Three series of ambiguity points located at the intersections of zero hyperplanes with hyperplanes of poles in any variable. They have the following coordinates: series $A^{++}: s=+\frac{2 m+1}{2}, t=+\frac{2 n+1}{2}$; series $A^{+-}: s=+\frac{2 m+1}{2}, t=-\frac{2 n+1}{2} \quad(m \geq n)$; series $A^{-+}: s=-\frac{2 m+1}{2}, t=+\frac{2 n+1}{2} \quad(m \leq n)$.

Let us consider the behavior of the amplitude $A(s, t)$ in the layers $B_{t}\{t \in \mathbf{R}, s \in \mathbf{C},|s|<\infty\}$ with $t \neq k+1 / 2$, were $k$ is integer. The only singularities of the amplitude in such layers are poles in the variable $s$.

Note that, beginning with some $n$, there is always a zero between two poles of $A(s, t)$. As the contours $C_{n}$ in the complex plane $s$, we take a system of circles (centered at the coordinate origin) passing through zeroes of the amplitude. It can be shown that everywhere on this system of contours, except a narrow sector in a vicinity of the real positive axis, the amplitude $A(s, t)$ has the Regge type asymptotics $\left(\sim s^{\frac{1}{2}+t}\right)$. In a vicinity of the real axis, the asymptotics is controlled by the presence of a zero.

In the terminology of Sec. 5 , in the layers ${ }^{8}$

$$
B_{t}\{t \in(n-1 / 2, n+1 / 2) ; \quad n=0,1, \ldots\}
$$

the amplitude $A(s, t)$ is an $n$-bounded function of the complex variable $s$ and of one real parameter $t$.

In the layers

$$
B_{t}\{t \in(-n-1 / 2,-n+1 / 2) ; \quad n=1,2, \ldots\}
$$

the amplitude has a decreasing asymptotics.

The residues of $A(s, t)$ at poles in $s$ are the same in all of the layers $B_{t}$ since pole positions do not depend on $t$. In the case where the point under consideration is not an ambiguity point, we have the following relations:

$$
r_{n}(t) \equiv R e s_{s=n+\frac{1}{2}} \frac{\Gamma\left(\frac{1}{2}-s\right) \Gamma\left(\frac{1}{2}-t\right)}{\Gamma(-s-t)}=\frac{1}{n !}\left(\frac{1}{2}+t\right) \cdots\left(\frac{1}{2}+t+n\right) \equiv \frac{1}{n !}\left(t+\frac{1}{2}\right)_{(n+1)},
$$

where $\left(t+\frac{1}{2}\right)_{(n+1)}$ is the so-called Pochhammer symbol (shifted factorial).

For example, let us construct the Cauchy expansion of $A(s, t)$ for $t \in(-3 / 2,-1 / 2)$. In this layer, $A(s, t)$ grows not faster then $s^{0-\epsilon}, \epsilon>0$, and there is no need in correcting polynomials. The Cauchy expansion looks as follows:

$$
A(s, t)=\sum_{n=0}^{\infty} \frac{1}{n !} \frac{\left(t+\frac{1}{2}\right)_{(n+1)}}{\left(s-n-\frac{1}{2}\right)}, \quad t \in(-3 / 2,-1 / 2) .
$$

Note that since the asymptotic becomes "softer" at large negative $t$, expansion (37) is also valid for any $t<-1 / 2$ (except the values corresponding to coordinates of the ambiguity points $t=-(2 k+1) / 2(k=0,1, \ldots)$, where the expansion makes no sense $\left.{ }^{9}\right)$.

In the layer $B_{t}\left\{t \in\left(-\frac{1}{2}, \frac{1}{2}\right)\right\}$, the amplitude $A(s, t)$ grows slower than a linear function of $s$, and thus in our Cauchy expansion we have to account the correcting polynomials of zero degree. Thus, we obtain the following expansion:

$$
A(s, t)=A(0, t)+\sum_{n=0}^{\infty} \frac{1}{n !}\left(\frac{\left(t+\frac{1}{2}\right)_{(n+1)}}{s-n-\frac{1}{2}}+\frac{\left(t+\frac{1}{2}\right)_{(n+1)}}{n+\frac{1}{2}}\right), \quad t \in(-1 / 2,1 / 2) .
$$

The technique of Cauchy forms allows us to represent a meromorphic function of two complex variables as a converging series of pole (in one variable) contributions; the convergence is uniform in both variables. This gives us the possibility to obtain two types of conditions on $A(s, t)$ : collapse conditions of superfluous degrees of the correcting polynomials and the bootstrap equations.

\footnotetext{
${ }^{8}$ Considering the example of the $B$-function, we use the natural brief notation for layers.

${ }^{9}$ In what follows, we do not mention this condition.
} 


\section{Collapse conditions and Bootstrap for Pochhammer symbols}

Collapse conditions on the regular part of the amplitude appear when we pass from the layer where the amplitude has an increasing asymptotics to another one, where the asymptotic regime is weaker. Expansion (38) for $A(s, t)$ in the layer $B_{t}\{t \in(-1 / 2,1 / 2)\}$ is also valid for $t<-1 / 2$. When $t$ crosses the boundary value $t=-1 / 2$ corresponding to the change of the asymptotic regime, the series of correcting polynomials can be summed independently:

$$
\sum_{n=0}^{\infty} h_{n}^{[0]}(t)=\sum_{n=0}^{\infty} \frac{1}{n !}\left(\frac{\left(t+\frac{1}{2}\right)_{(n+1)}}{n+\frac{1}{2}}\right)=-\frac{\Gamma\left(\frac{1}{2}-t\right) \Gamma\left(\frac{1}{2}\right)}{\Gamma(-t)}=-A(0, t), \quad t<-\frac{1}{2},
$$

and expansion (38) coincides with (37).

The bootstrap equations arise naturally from the requirement that the Cauchy expansion in one variable in some layer coincides with the expansion in the cross-conjugated variable (i.e., in the perpendicular layer) in the domain of intersection of these two layers. For example, expansion (38) is valid for the amplitude $A(s, t)$ in the layer $B_{t}\{t \in(-1 / 2,1 / 2)\}$. A similar expansion can be written in the layer $B_{s}\{s \in(-1 / 2,1 / 2)\}$ :

$$
A(s, t)=A(s, 0)+\sum_{n=0}^{\infty}\left(\frac{\rho_{n}(s)}{t-n-\frac{1}{2}}+\frac{\rho_{n}(s)}{n+\frac{1}{2}}\right)
$$

where $\rho_{n}(s) \equiv \frac{1}{n !}\left(s+\frac{1}{2}\right)_{(n+1)}$. These two expansions must coincide in the square, which is the intersection of two layers. Hence, in the domain $(s \sim 0, t \sim 0)$ the following condition must be satisfied:

$$
A(0, t)=A(s, 0)+\sum_{n=0}^{\infty}\left(\frac{\rho_{n}(s)}{t-n-\frac{1}{2}}+\frac{\rho_{n}(s)}{n+\frac{1}{2}}\right)-\sum_{n=0}^{\infty}\left(\frac{r_{n}(t)}{s-n-\frac{1}{2}}+\frac{r_{n}(t)}{n+\frac{1}{2}}\right) \equiv A(s, 0)+\Psi(s, t) .
$$

In some vicinity of the point $(0,0)$, the function $\Psi(s, t)$ is analytic since the corresponding series converge uniformly; thus, this function is completely determined by coefficients of its Taylor expansion at this point. Let us differentiate both parts of Eq. (40) with respect to $t$ :

$$
\frac{\partial A(0, t)}{\partial t}=\frac{\partial \Psi(s, t)}{\partial t} \quad(s \sim 0, t \sim 0)
$$

The left-hand side of this equality depends only on one variable $t$. This means that the dependence of $\frac{\partial \Psi(s, t)}{\partial t}$ on the second variable is purely fictitious. Thus, one may assign to $s$ any value from the domain $s \sim 0$ to compute the right-hand side of this equality. This allows us to determine the regular part of the amplitude up to one arbitrary constant $A(0,0)$.

These considerations allow us to rewrite (40) in the form of two conditions on the regular part of the amplitude plus an infinite system of consistency conditions:

$$
\begin{gathered}
\frac{\partial A(0, t)}{\partial t}=\left.\frac{\partial \Psi(s, t)}{\partial t}\right|_{s=0} \quad(t \sim 0), \\
\frac{\partial A(s, 0)}{\partial s}=-\left.\frac{\partial \Psi(s, t)}{\partial s}\right|_{t=0} \quad(s \sim 0), \\
\left.\frac{\partial^{k+p+2}}{\partial s^{k+1} \partial t^{p+1}} \Psi(s, t)\right|_{s=0, t=0}=0 \quad \text { for any } \quad k, p=0,1, \ldots .
\end{gathered}
$$

The consistency conditions express the fact that, in some vicinity of the point $(0,0)$, the derivative of $\Psi(s, t)$ with respect to any variable does not depend on the cross-conjugated variable.

Note that in this example the full symmetry between the variables $s$ and $t$ allows us to restrict our analysis of consistency conditions to the case $k>p$.

Such systems of conditions are called bootstrap equations. They represent nontrivial relations between resonance parameters (pole positions and residue values) of the function under consideration. In the present example, the pole position does not depend on the cross-channel variable. In this case, the system of bootstrap equations leads to an infinite set of relations for the values of residues (Pochhammer symbols). 
For example, let us consider the identity for Pochhammer symbols following from (43) with $k=1, p=0$ :

$$
\left\{\sum_{n=0}^{\infty} \frac{(-1) \rho_{n}^{(2)}(s)}{\left(t-n-\frac{1}{2}\right)^{2}}-\sum_{n=0}^{\infty} \frac{(-1)^{2} 2 ! r_{n}^{(1)}(s)}{\left(s-n-\frac{1}{2}\right)^{3}}\right\}_{s=0, t=0}=0
$$

One can easily show that the following equalities are valid for an arbitrary order derivative of the residue:

$$
\begin{gathered}
r_{n}^{(p)}(t)=0 \quad(p>n+1) ; \\
r_{n}^{(p)}(t)=\underbrace{\sum_{i_{1}=0}^{n} \sum_{i_{2}=0}^{i_{1}-1} \ldots \sum_{i_{p}=0}^{i_{p-1}-1}}_{i_{1}>i_{2} \ldots>i_{p}} \frac{p ! r_{n}(t)}{\left(\frac{1}{2}+i_{1}+t\right) \ldots\left(\frac{1}{2}+i_{p}+t\right)}, \quad p \leq n+1 .
\end{gathered}
$$

This allows us to rewrite relation (44) in the following way:

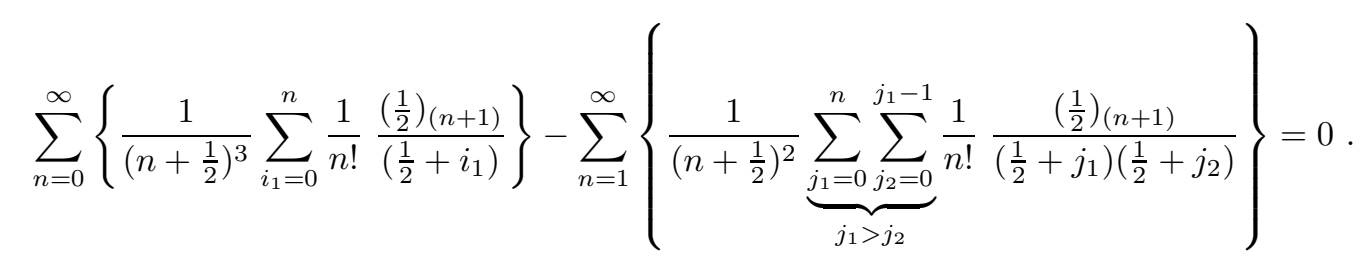

One can note that already the first consistency condition provides us with a highly nontrivial identity for Pochhammer symbols. The subsequent conditions lead to even more complicated identities. These identities reflect special properties of residues, which ensure the existence of a solution of the bootstrap system.

\section{Bootstrap SYSTEM IS OVERDETERMINED}

It is obvious that the system of bootstrap equations is probably overdetermined. The following question arises immediately: how to pick out a complete subsystem or to indicate which equations are obviously unnecessary (related)?

In addition to the system of bootstrap equations, we have at our disposal the collapse conditions of superfluous correcting polynomials in layers with a softer asymptotic behavior. Let us show how to use the collapse conditions and bootstrap in one layer in obtaining some of the bootstrap conditions in another layer.

In the three intersecting layers, $B_{t}\{t \in(-1 / 2,+1 / 2)\}, B_{t}\{t \in(-3 / 2,-1 / 2)\}$, and $B_{s}\{s \in(-3 / 2,-1 / 2)\}$, the following Cauchy expansions for the amplitude $A(s, t)$ are valid:

$$
\begin{gathered}
A(s, t)=A(0, t)+\sum_{n=0}^{\infty}\left(\frac{r_{n}(t)}{s-n-\frac{1}{2}}+\frac{r_{n}(t)}{n+\frac{1}{2}}\right), \quad B_{t}\{t \in(-1 / 2,+1 / 2)\} \\
A(s, t)=\sum_{n=0}^{\infty} \frac{r_{n}(t)}{s-n-\frac{1}{2}}, \quad B_{t}\{t \in(-3 / 2,-1 / 2)\}
\end{gathered}
$$

and

$$
A(s, t)=\sum_{n=0}^{\infty} \frac{\rho_{n}(s)}{t-n-\frac{1}{2}}, \quad B_{s}\{s \in(-3 / 2,-1 / 2)\} .
$$

We require that the corresponding expansions in the intersection domains of each of the two layers $B_{t}$ with the layer $B_{s}$ represent the same meromorphic function. This allows us to obtain the following bootstrap conditions:

$$
A(0, t)=\sum_{n=0}^{\infty} \frac{\rho_{n}(s)}{t-n-\frac{1}{2}}-\sum_{n=0}^{\infty}\left(\frac{r_{n}(t)}{s-n-\frac{1}{2}}+\frac{r_{n}(t)}{n+\frac{1}{2}}\right) \equiv \Psi_{1}(s, t) \quad(s \sim-1, t \sim 0)
$$

and

$$
0=\sum_{n=0}^{\infty} \frac{\rho_{n}(s)}{t-n-\frac{1}{2}}-\sum_{n=0}^{\infty} \frac{r_{n}(t)}{s-n-\frac{1}{2}} \equiv \Psi_{2}(s, t) \quad(s \sim-1, t \sim-1) .
$$


Using the same reasoning as in the previous section, we rewrite relations (49) as a system of the following conditions:

$$
A(0, t)=\Psi_{1}(-1, t),\left.\quad \frac{\partial^{k+p+1}}{\partial s^{k+1} \partial t^{p}} \Psi_{1}(s, t)\right|_{s=-1, t=0}=0 \quad \text { for any } k, p=0,1, \ldots
$$

The first of the above conditions gives us an explicit expression for the regular part of the amplitude, while the second condition reflects the independence of $\Psi_{1}(s, t)$ of the argument $s$.

Condition (50) can be rewritten in a similar form:

$$
\left.\frac{\partial^{k+p}}{\partial s^{k} \partial t^{p}} \Psi_{2}(s, t)\right|_{s=-1, t=-1}=0 \text { for any } k, p=0,1, \ldots
$$

The explicit expression for $A(0, t)$, which is valid in a vicinity of $t=0$, can be analytically continued without any obstruction to the domain of negative $t$, where the corresponding series converge very well.

Expansion (46) is also valid for $t<-\frac{1}{2}$. The requirement that expansions (46) and (47) must coincide for these values of $t$ implies the following collapse condition:

$$
A(0, t)=-\sum_{n=0}^{\infty} \frac{r_{n}(t)}{n+\frac{1}{2}}, \quad t<-\frac{1}{2} .
$$

This expression for the regular part of the amplitude must coincide with the analytic continuation of the expression obtained from (49) to the domain $t<-\frac{1}{2}$. In particular, this means that

$$
\Psi_{1}(-1, t)=-\sum_{n=0}^{\infty} \frac{r_{n}(t)}{n+\frac{1}{2}} \quad(s \sim-1, t \sim-1)
$$

As usual, let us rewrite the above relation as a condition on coefficients of the power series expansion in a vicinity of $(s=-1, t=-1)$ :

$$
\left.\frac{\partial^{p}}{\partial t^{p}} \Psi_{1}(-1, t)\right|_{t=-1}=-\left.\sum_{n=0}^{\infty} \frac{r_{n}^{(p)}(t)}{n+\frac{1}{2}}\right|_{t=-1}
$$

Using the explicit expression for $\Psi_{1}$, we obtain the relation

$$
\left\{\sum_{n=0}^{\infty} \frac{p !(-1)^{p} \rho_{n}(-1)}{\left(t-n+\frac{1}{2}\right)^{p+1}}-\sum_{n=0}^{\infty}\left(\frac{r_{n}^{(p)}(t)}{-1-n-\frac{1}{2}}+\frac{r_{n}^{(p)}(t)}{n+\frac{1}{2}}\right)\right\}_{t=-1}=-\left.\sum_{n=0}^{\infty} \frac{r_{n}^{(p)}(t)}{n+\frac{1}{2}}\right|_{t=-1}
$$

Collecting similar terms, we see that this condition coincides with (51) for all $p$ if $k=0$.

Thus, the considered example of three layers strengthens our confidence that the system consisting of bootstrap equations and collapse conditions is overdetermined. A share of information concerning bootstrap in lower layers is contained in the bootstrap equations for upper layers and in the collapse conditions for transitions from upper to lower layers.

\section{Numerical test of the CONVERGEnCE RATE}

Numerical testing of the convergence rate of the series in $n$ in expression (45) is of real interest. The reason is as follows: one of the ways to check theoretical predictions for dual models consists of saturating of identities (the so-called sum rules) quite similar to (45) with finite number of resonances.

Unfortunately, the up-to-date information on the hadron spectrum is far from being exhaustive (especially in the region $M>2 \mathrm{GeV}$ ). This means that only those sum rules that converge rapidly enough can undergo experimental verification. For this reason, it is extremely instructive to learn how to pick these identities out of the infinite system of bootstrap and collapse conditions. In this section, treating the example of the string amplitude discussed in Sec. 7, we suggest a possible approach to this problem in a realistic situation. 
Let us perform a numerical test of the following system of identities for the residues $r_{n}(t)$ and $\rho_{n}(s)$ obtained from the consistency conditions (41):

$$
\left\{\sum_{n=0}^{\infty} \frac{(-1)^{p+1}(p+1) ! \rho_{n}^{(k+1)}(s)}{\left(t-n-\frac{1}{2}\right)^{p+2}}\right\}_{s=0, t=0}-\left\{\sum_{n=0}^{\infty} \frac{(-1)^{k+1}(k+1) ! r_{n}^{(p+1)}(s)}{\left(s-n-\frac{1}{2}\right)^{k+2}}\right\}_{s=0, t=0}=0
$$

where $k, p \in \mathbf{N}, k>p$. The condition $k>p$ originates from the symmetry of the spectrum in $s$ and $t$.

First of all, we have to define a quantity which would allow us to characterize the precision of saturation of the sum rule (52) after taking into account a finite number of items. This can be done in the standard way, but in the current example the procedure of calculation can be sufficiently simplified. However, this point needs some comments since such simplifications are not always possible in realistic situations encountered in the field theory.

In expression (52), we deal with the difference of two absolutely convergent numerical series. Taking into account the symmetry of the spectrum, it seems natural to consider the difference of the contributions from $t$ and $s$-channel poles at every step of the computation. For the few first poles, this contribution has a definite sign (it is positive in the case $k>p$ ), but, beginning with some number $N_{+}(k, p)$, which depends on $k$ and $p$, the sign of the difference changes. Thus, the convergence of the series to zero is provided by the negative contribution of a large number of distant poles. This contribution compensates gradually the positive contribution of the first few poles. As a convergence characteristic, we choose the ratio

$$
D \equiv \frac{\Delta S(N)}{S_{+}}
$$

where $\Delta S(N)$ is the discrepancy which remains after the consideration of $2 N$ poles ( $N$ poles in the $s$-channel and $N$ poles in the $t$-channel) and $S_{+}$is the sum of all positive contributions (corresponding to a finite number of initial terms of the series of differences ${ }^{10}$ ). In terms of $D$, we can describe the convergence rate of the pole contribution series in the sum rules (52). To reduce the relative discrepancy significantly, it is sufficient to consider a small number of poles in rapidly converging sum rules.

The dependence of the relative discrepancy on the number of poles taken into account for the first three sum rules from system (52) is shown in the figure.

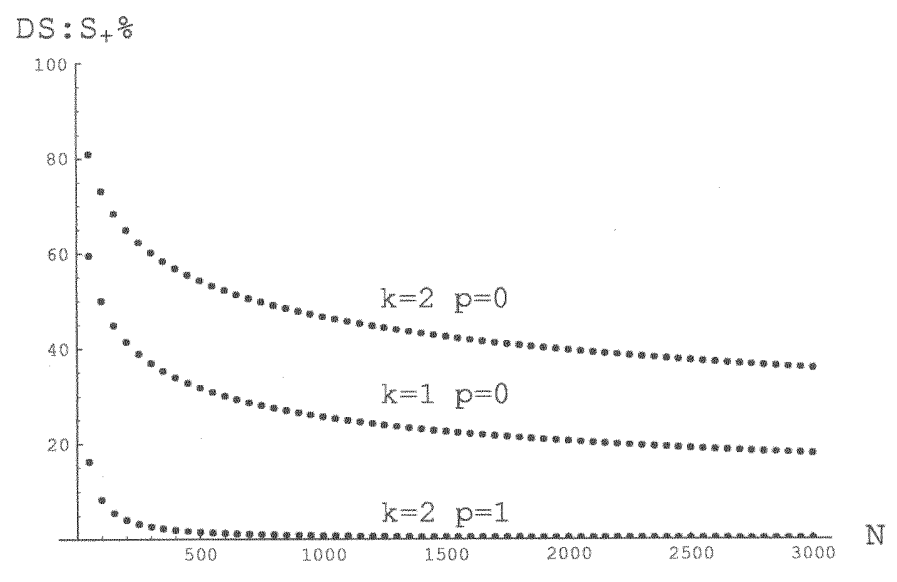

FIG. 1

The sum rule with $k=2, p=1$ converges sufficiently fast. Taking into account the first 100 poles, ${ }^{11}$ the relative discrepancy equals approximately $8 \%$ (1\% accuracy can be attained after the account of 700 poles). Thus, from this condition we can obtain a sufficiently good relation between the residues at the first 100 poles. The identities with $k=1, p=0$ and $k=2, p=0$ are not suitable for this purpose. The sum rule with $k=1, p=0$ converges much slower; the consideration of 3000 terms gives us $18 \%$ discrepancy, and to reduce it to $9 \%$, one needs to take into account more than 22000 terms. The sum rule with $k=2, p=0$ converges even more slowly.

\footnotetext{
${ }^{10}$ Of course, it only makes sense to consider $N>N_{+}$.

${ }^{11}$ In each channel.
} 
The sum rules with best convergence are those with $p=k-1$ at large values of $k$. One can expect that for large $k$, these sum rules are saturated rapidly.

It should be taken into account that $r_{n}^{(p)}(t)=0$ for $p>n+1$, hence the first poles do not contribute. Thus, these sum rules can serve as a source of relatively precise relations between parameters of several resonances with $n>p$.

The considered example allows us to understand in a qualitative way the main properties of constructions arising from the bootstrap conditions. In a realistic situation, only parameters of the few lightest resonances are known. It is even more surprising that, as shown in [6, 7] and [10], some of bootstrap restrictions are well saturated with available experimental data and provide a theoretical explanation to some phenomenological relations. This circumstance leads us to the idea that the $B$-function gives one a reasonable description only for the "tail" of the resonance spectrum. Parameters of the lower states are governed mostly by dynamical properties such as chiral symmetry.

\section{Conclusion}

The examples considered above show that the method of Cauchy forms is a useful tool for deriving relations between parameters of polynomially bounded meromorphic functions of two complex variables. It is easy to understand that, after the corresponding formulation of the analyticity conditions, this method can be in principle applied to the case of more variables. But the practical advantage of this approach to the study of inelastic amplitudes could hardly be notable. Even in the case of the simplest inelastic process $2 \rightarrow 3$, one needs five independent variables. This leads to extremely bulky expressions. A more powerful technique is necessary to allow us to compactify the notation.

The case of binary processes is interesting since it allows one to obtain relations between spectrum parameters determined by the requirements of correctness of the perturbative scheme of calculation the $S$-matrix. As was already mentioned, many of these relations are in excellent agreement with experimental data. This shows that even such a complicated construction as an effective field theory can be successfully applied to data analysis.

We are grateful to A. A. Andrianov, A. N. Vasiliev, M. I. Vyazovski, M. V. Polyakov, V. V. Sukhanov, H. Nielsen, P. Osland, V. Cheianov, and G. Schechter for fruitful discussions of various problems associated with the concept of an effective field theory.

This research was supported by INTAS (2000, project 587), the Russian Foundation for Basic Research (grant 01-02-17152), the Ministry of Education of Russia (E00-3.3-208), by the program "Universities of Russia" (UR.02.01.001), and by Meltzers Høyskolefond, Studentprosjektstipend 2002.

Translated by A. Vereshagin, V. Vereshagin, and K. Semenov-Tian-Shansky.

\section{REFERENCES}

1. S. Weinberg, Phys. Rev., 133, B1318 (1964); ibid., 134, B882 (1964); ibid., 135, B1049 (1964); ibid., 138, B988 (1965); ibid., 181, 1893 (1969).

2. Y. V. Novozhilov, Introduction to the Theory of Elementary Particles [in Russian], Moscow (1972).

3. J. B. Rumer and A. I. Fet, Group Theory and Quantum Fields [in Russian], Moscow (1977).

4. S. Weinberg, The Quantum Theory of Fields, Vols. 1-3, Cambridge University Press, Cambridge (2000).

5. S. Weinberg, Physica A, 96, 327 (1979).

6. V. Vereshagin, Phys. Rev., D55, 5349 (1997).

7. A. Vereshagin and V. Vereshagin, Phys. Rev., D59, 016002 (2000).

8. J. C. Collins, Renormalization, Cambridge University Press, Cambridge (1984).

9. P. H. Frampton, Dual Resonance Models and Superstrings, World Scientific (1986).

10. A. Vereshagin, $\pi N$ Newsletter, 16, 426 (2002).

11. S. Weinberg, Phys. Rev., 130, 776 (1963); ibid., 131, 440 (1963); ibid., 133, B232 (1964); M. Scadron and S. Weinberg, Phys. Rev., 133, B1589 (1964); M. Scadron, S. Weinberg, and J. Wright, Phys. Rev., 135, B202 (1964).

12. M. L. Nekrasov, Preprint IHEP 2001-9; e-archive hep-ph/0102283 (2001).

13. B. A. Kniehl and A. Sirlin, Phys. Letters, B530, 129 (2002).

14. M. Veltman, Physica, 29, 186 (1963).

15. B. V. Shabat, Introduction to the Complex Analysis [in Russian], Moscow (1969).

16. C. Lovelace, Phys. Letters, 28B, 264 (1968).

17. G. Veneziano, Nuovo Cimento, A57, 190 (1968). 